\title{
Mating system estimation via the one pollen parent model with the progeny array as the unit of observation
}

\author{
Daniel J. Schoen
}

Department of Biology, McGill University, 1205 Avenue Docteur Penfield, Montréal, Québec H3A 1B1, Canada.

\begin{abstract}
A method is presented for obtaining parameter estimates of the one pollen parent model using the progeny array as the unit of observation. The method is more direct and computationally simpler than that previously described by Schoen and Clegg (1984). It is shown that for a fixed total sample size the optimal number of progeny per family for the estimation of parental fixation index and selfing rate ranges from 3 to 9 , and depends on the level of inbreeding in the population.
\end{abstract}

\section{INTRODUCTION}

The mating system may strongly influence the amount and pattern of genetic variation in populations and thereby help determine the tempo and mode of evolutionary change. Accurate descriptions of the mating system, therefore, are frequently sought by population biologists. With the advent of electrophoresis as a tool for detecting single gene variation in natural populations, the number of studies reporting estimates of mating system parameters has increased substantially (see Schemske and Lande, 1985 for a partial list), and several mating system estimation models which differ as to their assumptions about the mating process have now been proposed (reviewed in Clegg, 1980; Ritland, 1983; Brown, Barrett, and Moran, 1985). The one pollen parent model, in contrast to the classical mixed mating model, assumes that outcrossed progeny within the family of a maternal parent practising mixed selfing and outcrossing are derived from pollination with a single paternal parent genotype. This may occur when the pollen deposited on the stigma(s) of the maternal parent consists of contributions from one plant (Schoen and Clegg, 1984, 1986). This type of mating system may characterize species with "packaged pollen" (e.g., members of the Orchidaceae, Asclepiadaceae, and Onagraceae). Moreover, there is evidence that the one pollen parent model accurately describes the mating system when families of seed are derived from single fruits within maternal parents in the species Ipomoea purpurea (Ennos, 1981; Schoen, 1985; Schoen and Clegg, 1986), and it may be applicable in other plants where seed families are derived entirely from single fruits within maternal parents.

This paper extends mating system estimation based on the one pollen parent model to treat the progeny array as the unit of observation (Ritland, 1986). This extension renders estimation of the model parameters and their variances more direct and computationally simpler than the procedure outlined by Schoen and Clegg (1984). For a more detailed discussion of the one pollen parent model, the reader should refer to Schoen and Clegg (1984, 1986).

A second objective of this paper is to provide information on optimal gene frequencies and progeny sizes for the estimation of parameters of the one-pollen parent model when allele frequencies are not close to 0 or 1 . Such information is useful in designing efficient sampling programs (Brown, 1975).

\section{METHOD OF ESTIMATION}

\section{Notation for the one pollen parent model}

The one pollen parent model is described below for a diallelic locus (with genotypes $A A, A a$, and $a a)$ by adopting the notation and approach of Ritland (1986) for the mixed mating model. Thus the model describes how the array of genotype 
frequencies in the offspring generation,

$$
g=\left(\begin{array}{c}
g(A A) \\
g(A a) \\
g(a a)
\end{array}\right)
$$

is related to a three-dimensional matrix with elements representing the frequencies of genotypic transitions from maternal and paternal parents to offspring, where the rows $i$ of this matrix index progeny genotypes, and the columns $j$ and $k$ index maternal and paternal parent genotypes, respectively $(i, j, k=1$ for $A A, i, j, k=2$ for $A a$, and $i, j, k=3$ for $a a$ ) (table 1 ). Specifically, in the one pollen parent model, progeny genotypes are related to parent genotypes as

$$
\boldsymbol{g}=[(1-s) \boldsymbol{T}+s \boldsymbol{S}] \boldsymbol{f}^{\mathrm{m} \otimes \boldsymbol{f}^{\mathrm{p}}}
$$

where $1-s$ is the fraction of progeny in the population (and within each maternal parent family) derived from outcrossing of maternal genotypes to single random paternal parent genotypes, $s$ is the fraction of progeny derived from self-fertilization, and $\otimes$ denotes element by element multiplication. The three-dimensional matrices $\boldsymbol{T}$ and $\boldsymbol{S}$ with row and column elements $T_{i j k}$ and $S_{i j k}$ are defined as follows,

$$
\begin{aligned}
& j=1 \quad j=2 \quad j=3 \\
& k=1 \quad k=2 \quad k=3 \quad k=1 \quad k=2 \quad k=3 \quad k=1 \quad k=2 \quad k=3 \\
& \boldsymbol{T}=\left(\begin{array}{ccc|ccc|ccc}
1 & 1 / 2 & 0 & 1 / 2 & 1 / 4 & 0 & 0 & 0 & 0 \\
0 & 1 / 2 & 1 & 1 / 2 & 1 / 2 & 1 / 2 & 1 & 1 / 2 & 0 \\
0 & 0 & 0 & 0 & 1 / 4 & 1 / 2 & 0 & 1 / 2 & 1
\end{array}\right)
\end{aligned}
$$

and,

$$
\begin{aligned}
& j=1 \quad j=2 \quad j=3 \\
& k=1 \quad k=2 \quad k=3 \quad k=1 \quad k=2 \quad k=3 \quad k=1 \quad k=2 \quad k=3 \\
& S=\left(\begin{array}{lll|lll|lll}
1 & 1 & 1 & 1 / 4 & 1 / 4 & 1 / 4 & 0 & 0 & 0 \\
0 & 0 & 0 & 1 / 2 & 1 / 2 & 1 / 2 & 0 & 0 & 0 \\
0 & 0 & 0 & 1 / 4 & 1 / 4 & 1 / 4 & 1 & 1 & 1
\end{array}\right)
\end{aligned}
$$

where the elements of $\boldsymbol{T}$ are conditional probabilities of progeny genotypes $i$ given that maternal parent $j$ is outcrossed to paternal parent $k$, and the elements of $\boldsymbol{S}$ are conditional probabilities of progeny genotype $i$ given that the maternal parent within the mating type $j k$ is self-fertilized. The column vectors $\boldsymbol{f}^{\mathrm{m}}$ and $\boldsymbol{f}^{\mathrm{p}}$ with elements $f_{j}^{\mathrm{m}}$ and $f_{k}^{\mathrm{p}}$ are defined as $F f^{\mathrm{im}}+(1-F) f^{\mathrm{om}}$ and $F f^{\mathrm{ip}}+$ $(1-F) f^{\text {op }}$, respectively. $F$ denotes Wright's gene fixation index (Wright, 1969),

$$
f^{\mathrm{im}}=\left(\begin{array}{c}
u \\
0 \\
v
\end{array}\right), \quad f^{\mathrm{om}}=\left(\begin{array}{c}
u^{2} \\
2 u v \\
v^{2}
\end{array}\right),
$$

$$
f^{\mathrm{ip}}=\left(\begin{array}{c}
p \\
0 \\
q
\end{array}\right), \quad f^{\mathrm{op}}=\left(\begin{array}{c}
p^{2} \\
2 p q \\
q^{2}
\end{array}\right),
$$

and $u=1-v$ and $p=1-q$ denote the frequencies of the $A$ allele in the maternal and paternal parent pool, respectively; i.e., the vectors $f^{\mathrm{im}}$ and $f^{\mathrm{om}}$ contain genotypes frequencies among maternal parents with fixed alleles and with alleles not fixed, respectively, while the vectors $f^{\text {ip }}$ and $f^{\text {op }}$ contain genotypes frequencies among paternal parents with fixed alleles and with alleles not fixed. Sampled progeny arrays are denoted as $x_{y}(y=$ $1,2, \ldots, N)$ and have elements $x_{i y}$ corresponding to the observed number of progeny of genotype $i$ in array $y$, with $\sum x_{i y}=n_{y}$. The set of all $x_{y}$ vectors forms the matrix $\boldsymbol{X}$.

\section{Probability and likelihood of progeny array data}

The probability of observing segregation pattern $\boldsymbol{x}_{y}$ given maternal parent $j$ and paternal parent $k$ is

$$
\operatorname{Pr}_{y j k}=\left(\frac{n_{y} !}{\prod_{i} x_{i} !}\right) \prod_{i}\left[s S_{i j k}+(1-s) T_{i j k}\right] x_{i y},
$$

and by accounting for the possibility of drawing all maternal parents $j$ and paternal parents $k$, the overall probability of array $y$ is

$$
\operatorname{Pr}_{y}=\sum_{y} \sum_{k} f_{j}^{\mathrm{m}} f_{k}^{\mathrm{p}} \operatorname{Pr}_{y j k} \text {. }
$$

Letting $P_{i j k}$ denote the probability of progeny genotype $i$ given maternal parent $j$ and paternal parent $k$, where

$$
P_{i j k}=s S_{i j k}+(1-s) T_{i j k},
$$

the likelihood of progeny array $\boldsymbol{x}_{\boldsymbol{y}}$ given maternal parent $j$ and paternal parent $k$ can be written as

$$
L_{y j k}=\prod_{i}\left(P_{i j k}\right)^{x_{i k}}
$$

Accounting for alternative maternal and paternal parentage, the likelihood of progeny array $\boldsymbol{x}_{\boldsymbol{y}}$ is

$$
\begin{aligned}
L_{y}^{\mathrm{a}}= & \sum_{j} \sum_{k}\left[F f_{j}^{\mathrm{im}}+(1-F) f_{j}^{\mathrm{om}}\right] \\
& \times\left[F f_{k}^{\mathrm{ip}}+(1-F) f_{k}^{\mathrm{op}}\right] L_{y j k} \\
= & \sum_{j} \sum_{k} f_{j}^{\mathrm{m}} f_{k}^{\mathrm{p}} L_{y j k} .
\end{aligned}
$$

The likelihood of the total sample of progeny arrays is

$$
L^{t}(\boldsymbol{X})=\prod_{y} L_{y}^{\mathrm{a}}
$$


Table 1 Matrix representation of the one pollen parent model with rows $i$ indexing progeny genotypes and columns $j$ and $k$ indexing maternal and paternal parent genotypes, respectively. Elements are frequencies of genotypic transitions from mating types to progeny genotypes. Outcrossing is assumed to occur with probability $1-s$, and selfing with probability $s$

\begin{tabular}{|c|c|c|c|}
\hline & & $j=1$ & \\
\hline & $k=1$ & $k=2$ & $k=3$ \\
\hline$i=1$ & $f(A A, A A \rightarrow A A)$ & $f(A A, A a \rightarrow A A)$ & $f(\boldsymbol{A} \boldsymbol{A}, a a \rightarrow A A$ \\
\hline$i=2$ & $f(A A, A A \rightarrow A a)$ & $f(A A, A a \rightarrow A a)$ & $f(A A, a a \rightarrow A a)$ \\
\hline \multirow[t]{3}{*}{$i=3$} & $f(A A, A A \rightarrow a a)$ & $f(A A, A a \rightarrow a a)$ & $f(A A, a a \rightarrow a a)$ \\
\hline & & $j=2$ & \\
\hline & $k=1$ & $k=2$ & $k=3$ \\
\hline$i=1$ & $f(A a, A A \rightarrow A A)$ & $f(A a, A a \rightarrow A A)$ & $f(A a, a a \rightarrow A A)$ \\
\hline$i=2$ & $f(A a, A A \rightarrow A a)$ & $f(A a, A a \rightarrow A a)$ & $f(A a, a a \rightarrow A a)$ \\
\hline \multirow[t]{3}{*}{$i=3$} & $f(A a, A A \rightarrow a a)$ & $f(A a, A a \rightarrow a a)$ & $f(A a, a a \rightarrow a a)$ \\
\hline & & $j=3$ & \\
\hline & $k=1$ & $k=2$ & $k=3$ \\
\hline$i=1$ & $f(a a, A A \rightarrow A A)$ & $f(a a, A a \rightarrow A A)$ & $f(a a, a a \rightarrow A A)$ \\
\hline$i=2$ & $f(a a, A A \rightarrow A a)$ & $f(a a, A a \rightarrow A a)$ & $f(a a, a a \rightarrow A a)$ \\
\hline$i=3$ & $f(a a, A A \rightarrow a a)$ & $f(a a, A a \rightarrow a a)$ & $f(a a, a a \rightarrow a a)$ \\
\hline
\end{tabular}

Note that the model assumes homogeneity of selfing rate and uniform probabilities of drawing a particular paternal parent genotype across maternal parents, which can also be interpreted as lack of spatial heterogeneity in pollen parent geotype frequencies.

\section{Method of finding the maximum likelihood estimates (MLE's) of the one-pollen parent model}

The procedure used below for finding the MLE's is based on the scoring method of Fisher (Rao, 1952). It is, however, also possible to find the MLE's using the expectation-maximization algorithm (Demster, Laird, and Rubin, 1977). For a comparison of the merits of different methods see Ritland (1986). The first derivatives of the log likelihood function for progeny array $x_{y}$ with respect to the model parameters $\boldsymbol{b}=(F, s, p, u)$ (i.e., the scores, denoted by $S_{y}^{b}$ ) are as follows:

$$
\begin{aligned}
& S_{y}^{\mathrm{F}}=\left\{\sum _ { j } \sum _ { k } \left\{f_{j}^{\mathrm{m}}\left[f_{k}^{\mathrm{ip}}-f_{k}^{\mathrm{op}}\right]\right.\right. \\
& \left.\left.+f_{k}^{\mathrm{p}}\left[f_{j}^{\mathrm{im}}-f_{j}^{\mathrm{om}}\right]\right\} L_{y j k}\right\} / L_{y}^{\mathrm{a}}, \\
& S_{y}^{\mathrm{s}}=\left\{\sum_{j} \sum_{k} f_{j}^{\mathrm{m}} f_{k}^{\mathrm{p}} L_{y j k} \sum_{i}\left(S_{i j k}-T_{i j k}\right) / P_{i j k}\right\} / L_{y}^{\mathrm{a}},
\end{aligned}
$$

$$
\begin{aligned}
& S_{y}^{\mathrm{p}}=\left\{\sum_{j} \sum_{k} f_{j}^{\mathrm{m}}\left[F f_{k}^{\mathrm{i}^{\prime}}+(1-F) f_{k}^{\mathrm{op}^{\prime}}\right] L_{y j k}\right\} / L_{y}^{\mathrm{a}}, \\
& S_{y}^{\mathrm{u}}=\left\{\sum_{j} \sum_{k} f_{k}^{\mathrm{p}}\left[F f_{j}^{\mathrm{im}^{\mathrm{m}^{\prime}}}+(1-F) f_{j}^{\mathrm{om}^{\prime}}\right] L_{y j k}\right\} / L_{y}^{\mathrm{a}},
\end{aligned}
$$

where $f_{k}^{\mathrm{ip} \mathbf{p}^{\prime}}$ and $f_{k}^{\mathrm{op}}$, are derivatives with respect to $p$ of the elements in $\boldsymbol{f}^{\mathrm{ip}}$ and $\boldsymbol{f}^{\mathrm{op}}$, and $f_{j}^{\mathrm{im} \mathrm{m}^{\prime}}$ and $f_{j}^{\text {om }}$ are derivatives with respect to $u$ of the elements in $\boldsymbol{f}^{\mathrm{im}}$ and $\boldsymbol{f}^{\mathrm{om}}$. Joint maximum likelihood estimates of the parameters $\boldsymbol{b}$ can be obtained by jointly iterating the four recursions

$$
b_{i}^{*}=b_{i}+\sum_{y}\left(S_{y}^{b_{i}} / I^{b_{i}}\right)
$$

for $i=1, \ldots, 4$, where

$$
I^{b_{i}}=\sum_{y}\left(S_{y}^{b_{i}}\right)^{2}
$$

A computer program which performs the above computations is available from the author on request.

\section{OPTIMAL ALLELE FREQUENCY AND PROGENY SIZE}

Asymptotic variances of the estimates in $\boldsymbol{b}$ were obtained by inverting the Fisher information matrix with elements $\mathrm{E}\left[\left(d \ln L / d b_{i}\right)\left(d \ln L / d b_{j}\right)\right]$. 
These elements can be calculated in terms of previous formulas (Ritland, 1986), i.e., the information matrix has elements which are equal to

$$
\sum_{x_{y}} \operatorname{Pr}_{y}^{\mathrm{a}} S_{y}^{\mathrm{b}_{i}} S_{y^{j}}^{b_{j}}
$$

where $\operatorname{Pr}_{y}^{\mathrm{a}}$ and the $S_{y}^{b_{i}}$ 's are as outlined above, and the summation is over all the possible classifications of $n_{y}$ progeny into genotypic classes $A A, A a$, and $a a$.

The variance of estimates of $F$ and $s$ per zygote, obtained by dividing the elements of the information matrix by $n_{y}$ before inverting it, are plotted against allele frequency in fig. 1. As in the case of the mixed mating model (Ritland, 1986), the best estimates of $F$ and $s$ are obtained for allele frequency $p=u=0.5$.

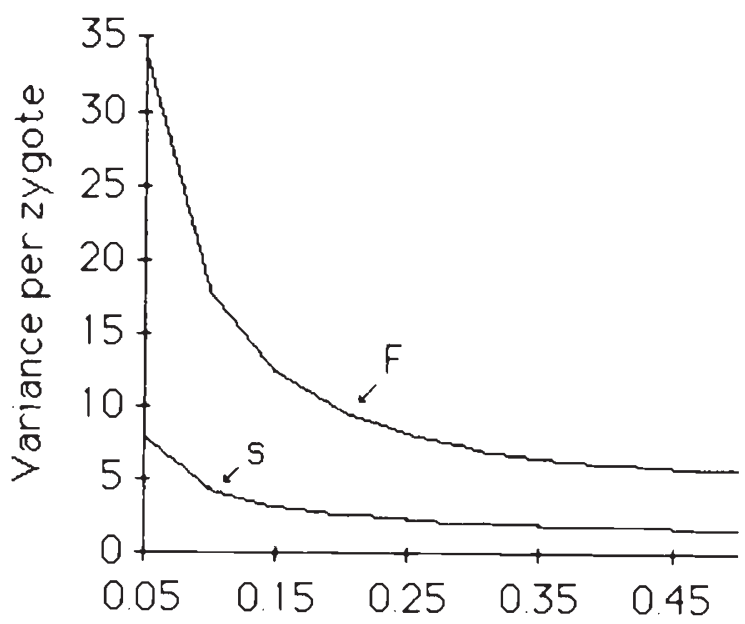

Allele frequency

Figure 1 Variance of estimates of the one pollen parent model per zygote as a function of allele frequency for a family size of 5 progeny and $F=s=0.5$.

Figs. 2(a)-(c) shows the variances of these esimates in relation to family size. When there is intermediate inbreeding $(F=s=0.5)$ and $p=u=$ 0.25 , the best estimates of $F$ are obtained for family sizes of 4 or 5 progeny. In outbreeding populations $(F=s=0.01)$, optimal family size increases to 8 or 9 progeny, while in inbreeding populations $(F=s=0 \cdot 9)$, optimal family size for estimation of $F$ decreases to 3 or 4 progeny. Optimal family size for the estimation of $s$ is always large.

\section{EXAMPLE OF ESTIMATION}

The routine outlined above was applied to data obtained as part of a study of the mating system
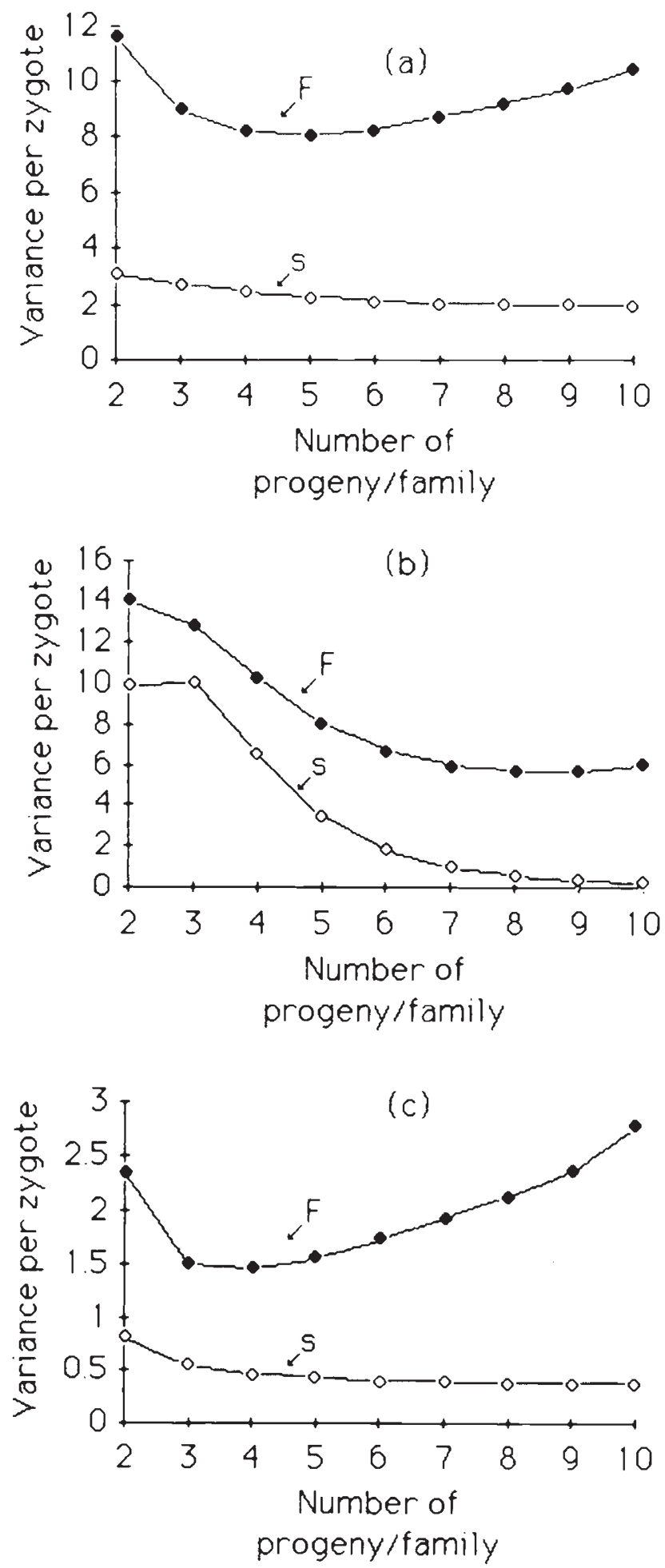

Figure 2 Variance of estimates of the one pollen parent model per zygote as a function of number of progeny per family (a) $F=s=0.5$, (b) $F=s=0.01$, and (c) $F=s=0.9$. Note $p=u=0.25$ in all three cases. 
of Ipomoea purpurea (Schoen and Clegg, 1985). Progeny arrays derived from single fruits within maternal parents, are listed in $\boldsymbol{X}$

$$
\begin{aligned}
\boldsymbol{X}= & \left(\begin{array}{llllllll}
000 & 000 & 000 & 000 & 000 & 000 & 000 & 000 \\
111 & 211 & 103 & 111 & 424 & 014 & 300 & 100 \\
333 & 233 & 341 & 333 & 020 & 430 & 144 & 344 \\
030 & 423 & 344 & 341 & 300 & 121 & 011 & 401 \\
114 & 021 & 100 & 103 & 123 & 122 & 222 & 023 \\
300 & 000 & 000 & 000 & 021 & 201 & 211 & 020
\end{array}\right)
\end{aligned}
$$

where the $i$ th row and $y$ th column of $\boldsymbol{X}$ give the number of progeny genotypes $A A, A a$, and $a a$ in the $y$ th family. The order of progeny arrays listed in $\boldsymbol{X}$ reflects three grouped collections from each of the two types of homozygous seed parents and the heterozygote, respectively, as outlined in the experimental design of Schoen and Clegg (1985). Table 2 shows the MLE's of the one-pollen parent model for 25 iterations when starting values for all parameters are set to $0 \cdot 5$. If some a priori knowledge of the parameter values is available, the number of iterations required for convergence can be reduced by starting the estimation routine with these.

Table 2 Parameter estimates, likelihoods, and iteration values for the current iteration when the one pollen parent model estimation procedure is applied to the progeny arrays in matrix $X$ (see text)

\begin{tabular}{llllll}
\hline$F$ & $s$ & $p$ & $u$ & $\log \left(L^{\mathrm{t}}\right)$ & Iteration \\
\hline 0.500 & 0.500 & 0.500 & 0.500 & -169.930 & 1 \\
0.238 & 0.519 & 0.599 & 0.372 & -166.554 & 2 \\
0.282 & 0.548 & 0.661 & 0.352 & -166.217 & 3 \\
0.269 & 0.563 & 0.680 & 0.339 & -166.149 & 4 \\
0.265 & 0.568 & 0.696 & 0.337 & -166.134 & 5 \\
0.260 & 0.577 & 0.703 & 0.333 & -166.130 & 10 \\
0.259 & 0.577 & 0.705 & 0.334 & -166.129 & 15 \\
0.259 & 0.577 & 0.705 & 0.334 & -165.129 & 25 \\
\hline
\end{tabular}

It is noteworthy that when this estimation procedure is applied to family data simulated according to the assumptions of the mixed mating model, i.e., with many rather than one pollen parent genotypes per family as described in Schoen and Clegg $(1984,1986)$, that estimates do not converge in some instances (Schoen, unpublished). In practice, such non-convergence might be interpreted as an indication of violation of the model assumption of one male parent among the outcrossed progeny of a family.

\section{CONCLUSION}

An algorithm has been presented for estimating the parameters of the one pollen parent model using the progeny array as the unit of observation. The method is more direct than that outlined by Schoen and Clegg (1984). The latter method was based on a two stage procedure which did not allow straightforward computation of the variances of the estimates (Clegg, Kahler, and Allard, 1978). This paper also reveals that optimal family sizes for the estimation of $F$ are similar to those reported by Ritland (1986) for the mixed mating model, i.e., family sizes are smaller than those adopted by many researchers.

At present, due to the small number of studies which have focussed on the problems the overall relevance of the one pollen parent model to mating system estimation in plants is not clear. Several studies suggest that the model may be most useful in situations where the progeny in arrays derive from the single fruits. For example, Schoen (1985) and Brown, Grant, and Pullen (1986) found evidence in Ipomoea purpurea and Glycine argyrea, respectively, that seeds derived from outcrossing within the same fruit on a plant were more likely to share paternal parentage than seeds of different fruits on a plant. In the latter case, it was estimated that 85 per cent of the cross-pollinated seed within a fruit shared the same male parent. Ritland (personal communication) reports a similar hierarchy of male parentage in Mimulus guttatus. Such a hierarchy suggests a role for the model in mating system estimation, but it remains to be seen how applicable the assumptions of this model will apply in general. It is hoped that the above-outlined presentation of the one pollen parent model will help to simplify its use in mating system estimation, and help to unify approaches to obtaining these estimates by putting the problem into the framework provided by Ritland (1986) for the mixed mating and effective selfing models.

\footnotetext{
Acknowledgements This research was supported by a grant from the Natural Sciences and Engineering Research Council of Canada. I thank Dr. Steven Stewart and Kermit Ritland for discussing this problem with me.
}

\section{REFERENCES}

BROWN, A. H. D. 1975. Efficient experimental designs for the estimation of genetic parameters in plant populations, Biometrics, 31, 145-160. 
BROWN, A. H. D., BARRETT, S. C. H. AND MORAN, G. F. 1985. Mating system estimation in forest trees: models, methods and meanings. In Gregorius, H.-R. (ed.) Population Genetics in Forestry, Springer-Verlag, Berlin, pp. 32-49.

BROWN, A. H. D., GRANT, J. E., AND PULLEN, R. 1986. Outcrossing and paternity in Glycine argyrea by paired fruit analysis. Biol. J. Linn. Soc., 29, 283-294.

CLEGG, M. T. 1980. Measuring plant mating systems. Bioscience, $30,814-818$

CLEGG, M. T., KAHLER, A. L. AND ALLARD, R. w. 1978. Estimation of life cycle components of selection in an experimental plant population. Genetics, 89, 765-792.

DEMSTER, A. P., LAIRD, L. M. AND RUBIN, D. B. 1977. Maximum likelihood from incomplete data via the EM algorithm. Jour. Roy. Stat. Soc., Ser. B, 39, 1-38.

ENNOS, R. A. 1981. Quantitative studies of the mating system in two sympatric species of Ipomoea (Convolvulaceae). Genetica, 57, 93-98.

RAO, C.R. 1952. Advanced Statistical Methods in Biometric Research. Chapman and Hall, New York.

RITLAND, K. 1983. Estimation of mating systems. In Tanksely, S. D. and Orton, T. J. (eds) Isozymes in Plant Genetics and Plant Breeding, part A, Elsevier Science Publishers, Amsterdam, pp. 289-302.
RITLAND, K. 1986. Joint maximum likelihood estimation of genetic and mating structure using open-pollinated progenies. Biometrics, 42, 25-43.

SCHEMSKE, D. W. AND LANDE, R. 1985. The evolution of self-fertilization and inbreeding depression in plants. II. Empirical observations. Evolution, 39, 41-52.

SCHOEN, D. J. 1985. Correlation between classes of mating events in two experimental plant populations. Heredity, 55, 381-385.

SCHOEN, D. J. AND CLEGG, M. T. 1984. Estimation of mating system parameters when outcrossing rates are correlated. Proc. Natl. Acad. Sci. U.S.A., 81, 5258-5262.

SCHOEN, D. J. AND CLEGG, M. T. 1985. The influence of flower color on outcrossing rate and male reproductive success in Ipomoea purpurea. Evolution, 39, 1242-1249.

SCHOEN, D. J. AND ClEGG, M. T. 1986. Monte Carlo studies of plant mating system estimation models: the one-pollen parent and mixed mating model. Genetics, 112, 927-945.

WRIGHT, S. 1969. Evolution and the Genetics of Populations. Vol. II. The Theory of Gene Frequencies. University of Chicago Press, Chicago. 